\title{
ULTRAVIOLET ASTRONOMY
}

\author{
R. WILSON \\ (Culham Laboratory, England)
}

\section{Introduction}

Ultraviolet astronomy is in a phase of very rapid development and any review of the new techniques involved is necessarily conditioned by the fact that most of the work is in a preparatory stage. This paper, therefore, will not be restricted to a discussion of those techniques which have undergone the ultimate test of their effectiveness, i.e., an actual space mission, but will include a consideration of those techniques which are still under development. Some omissions are therefore inevitable.

The presentation is divided into three categories: (a) the vehicle and its stabilisation (if any), (b) the optics, and (c) detectors. Category (a) presents the major new technological problems and contributes the greater part of the total cost. Experience in the other categories of optics and detectors had, of course, reached an advanced and sophisticated level at the time of the advent of the rocket, but new and difficult problems have been posed mainly by their operation in a space environment, but also by their use in the ultraviolet region of the spectrum.

\section{Vehicle Stabilisation}

Many of the technical problems posed by UV astronomy are common to all areas of space research, e.g., power supply, telemetry, etc., and are not dealt with here. However, new problems are posed by the fact that the space vehicle is used as a platform for the observation of remote objects, in contrast to most geophysical experiments where the vehicle is used as a probe. For astronomical studies it is therefore essential to know the attitude of the vehicle with time and for any detailed observations it is also necessary to control the attitude.

\section{A. UNSTABILISED SOUNDING ROCKETS}

The pioneering observations in UV astronomy were made from unstabilised sounding rockets in which the sky was scanned by the motion of the vehicle. The limited observing time per star therefore restricted the experiments to measurement of the UV flux in broad bands from the brightest early-type stars.

The first ultraviolet astronomical observations were secured in a rocket flight during

Perek (ed.), Highlights of Astronomy, 94-107. (C I.A.U. 
1955 by Byram et al. (1957) of N.R.L., which was followed by further observations by that group (Friedman, 1962; Chubb and Byram, 1963; Byram et al., 1964). The early observations were made with mechanical collimating devices feeding broad-band selective photomultipliers. These were later replaced by prime focus telescopes, feeding ionisation chambers operating in the range 1200-1500 $\AA$. The photometers were flown in the American Aerobee rocket with a viewing direction perpendicular to the rocket axis, a sky scan being obtained by the roll motion of the vehicle. Using the same rocket, Boggess $(1961,1964)$ has made observations in the $2000-3000 \AA$ region, using photoelectric photometers fed by Cassegrain telescopes; and Gulledge and Packer (1963) observed bands centred at $2985 \AA$ and $2100 \AA$ with respective widths of $52 \AA$ and $160 \AA$ by employing photomultipliers and interference filters. Observations with the British Skylark rocket have been made in the Southern hemisphere by Alexander et al. (1964) using a mechanical collimator to feed a selective photomultiplier giving a bandwidth of $\sim 400 \AA$ centred at $1950 \AA$. The first dispersing system was flown by Stecher and Milligan (1962) in the Aerobee rocket. The stellar radiation passed through a mechanical collimator onto a large plane grating, the deflected light then being focussed onto an entrance slit by two reflections and measured by a photomultiplier. The spin axis of the rocket was normal to the plane of dispersion and the stellar spectrum was scanned by the motion of the rocket. The operating spectral resolution was set at $\sim 100 \AA$, and observations were limited to stars brighter than about third magnitude.

\section{B. SCANNING SATELLITES}

The type of sky scan performed by unstabilised rockets can also be employed from satellites with a consequent increase in total observational time. Further, a reduction in the spin rates of the vehicle will result in a greater observational time per object, and by 'scanning satellites' is meant those vehicles for which some control of angular rates is exercised. This may result in either a slow but unpredictable scan of the sky or a controlled scan over the celestial sphere.

1964-83C: Smith (1967a) has flown a photometer in the American satellite 1964-83C and obtained several stellar observations with a pass band $\sim 200 \AA$ centred at $1376 \AA$. The satellite employed a form of magnetic stabilisation which allowed a slow scan of the sky. Because of the complexity of the dynamic effects this scan was not predictable and the attitude was determined from solar sensors and magnetometers followed by star recognition.

Cosmos-51: Dimov and Severny (1965) flew a photometer in the Soviet satellite Cosmos-51 and obtained observations with a pass-band of $\sim 300 \AA$ centred at $2700 \AA$, together with a simultaneous observation in the visible. A form of stabilisation allowed a slow scan of the sky but, as in the case of 1964-83C, the attitude was not predictable and attitude information was determined from ancillary sensors and star recognition.

Venus-3: Ultraviolet observations have been made by Kurt and Syunyaev (1967) 
from the Soviet deep-space probe Venus-3. The photometers used had wide fields in order to measure the sky background radiation in the spectral intervals $1050-1340 \AA$ and 1225-1340 $\AA$ using Geiger counters with appropriate gas and windows.

ESRO-TD1: This European satellite is under development and is planned to operate in a Sun-synchronous orbit. It will be Sun-stabilised with a roll control which maintains a rotational period equal to the orbital period, thus enabling experiments mounted in the appropriate axis to scan a great circle of the sky in each orbit and the precession of $\sim 1^{\circ}$ per day will allow a systematic coverage of the celestial sphere. This controlled kind of scan will therefore give attitude information, but this will still be supplemented by star recognition.

Two ultraviolet astronomical experiments are to be carried out in this satellite. A combined Edinburgh/Liège (Housiaux, 1965) package consists of a $25-\mathrm{cm}$ telescope feeding a photometer which will measure four pass-bands between $1250 \AA$ and $3000 \AA$ with bandwidths between $100 \AA$ and $200 \AA$. An infrared photometer is also included for measurements near $3 \mu$. The second ultraviolet experiment is being prepared by the Utrecht Group (De Jager et al., 1967); this employs a $22-25 \mathrm{~cm}$ telescope feeding a grating spectrometer with scanning exit slits and photomultipliers. In this case the experiment includes its own fine stabilisation device which selects a bright star in the field of view and counterrotates the telescope so as to take out the scanning motion of the satellite and thereby allow observation times per object to be substantially increased to $4 \mathrm{~min}$. This allows a much higher spectral resolution to be employed and the exit-slit widths are set at $1 \AA$, the spectrum being scanned in steps of $0.5 \AA$. It is expected that stellar spectra will be obtained down to magnitude 4 .

SAS: A small astronomical satellite is being developed by NASA (1967) to be launched in a Scout rocket. Present plans call for a gyro-stabilised spacecraft which can be controlled in three axes by magnetic torquing and allow a variation in the type of scan which can be performed. To date no ultraviolet experiment has been assigned to this type of vehicle, the first of which will carry an X-ray experiment.

\section{BALLOONS}

Although ultraviolet observations down to the Lyman limit of atomic hydrogen at $912 \AA$ are possible only from heights requiring rocket vehicles, observations from high-altitude balloons can extend the accessible spectrum down to $1900 \AA$. A group at the Geneva Observatory (Golay et al., 1967) has obtained low-resolution objective prism spectra using French balloons and a gondola equipped with spinning rotator stabiliser which allows a sweep of the sky at $0 \cdot 4^{\circ}$ per sec.

\section{STABILISED ROCKETS}

The full development of ultraviolet astronomy requires space vehicles which can be 
fully stabilised with respect to the object being observed. For a package consisting of a telescope and grating spectrometer, the degree of stabilisation can be determined by expressing the element of spectral resolution $\delta \lambda$ in terms of the angle $\delta \omega$ it subtends in the telescope field, thus:

$$
\delta \omega=\stackrel{G}{T} D \delta \lambda,
$$

where $G$ is the diameter of the grating, $D$ its angular dispersion, and $T$ the diameter of the telescope. Typical values are $G / T=0 \cdot 1$ and $D=25 \operatorname{arcsec}$ per $\AA$, giving $\delta \omega=2 \cdot 5$ $\operatorname{arcsec}$ for $\delta \lambda=1 \AA$. Whereas in the case of scanning systems the observational time per object is limited by the scanning rates, in the case of a fully stabilised system the limitation is imposed only by the trajectory time for a sounding rocket, and by occultational effects for a satellite.

A number of star-pointing stabilisation systems are currently under development for operation in sounding rockets, and these are summarised in Table 1.

The IACS (Inertial Attitude Control System) (NASA, 1967) is based on a 3-axis gyro-reference which is programmed before flight, actuation being achieved by a gas jet system. The STRAP (Stellar Tracking Rocket Attitude Position) and FACS (Fine Attitude Control System) (NASA, 1967) systems are based on the IACS but have additional star trackers which take over the control on acquisition and use an auxiliary low-thrust jet system to give higher pointing accuracies.

The U.K. Starling (Elliott Bros., 1967) system is based on a Moon-pointing control unit which is now in an advanced state of development. This unit will be used for offsetting to the desired star, at which point a star tracker will take over for the final acquisition. Actuation is by means of gas jets.

The German system being developed by Dornier to the requirements of the MaxPlanck Institute will use a spinning rocket, the axis being aligned at a required star from an inertial reference. The final acquisition will be made by means of a star sensor and gas jets will be used for actuation.

Of the control units described in Table 1 only the IACS and STRAP systems are currently operational and have undergone a number of successful flights, which have obtained new and important results in UV astronomy. The Princeton group (Morton and Spitzer, 1966; Morton, 1967) have used the IACS system on Aerobee rockets and obtained objective grating spectrograms of a number of bright stars in the range $1200-3000 \AA$ with spectral resolutions between 1-3 $\AA$. This rather good spectral resolution was achieved by attaching the experimental package to a large gyroscopic rotor which gave an additional fine stabilization in the direction of dispersion, the limit cycle being reduced to \pm 16 arcsec. Objective grating spectra have also been obtained in the 1600-3000 $\AA$ range with a resolution of about $10 \AA$ by Boggess (1967) using the IACS/Aerobee. The STRAP system has been flown on an Aerobee rocket by Stecher (1967), who employed a 13-inch telescope with a scanning 3-channel photoelectric spectrometer and obtained a number of stellar spectra over the range 1200 


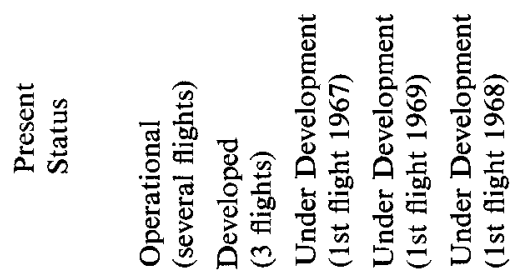

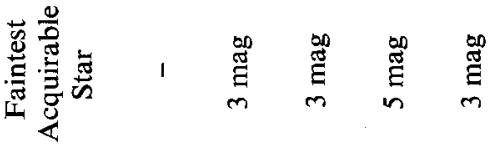

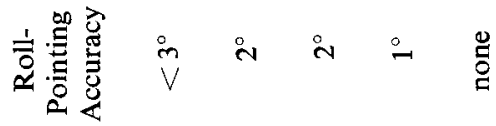

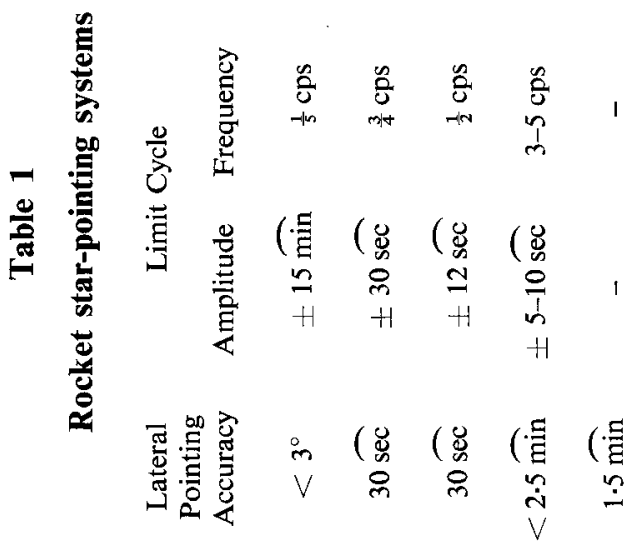

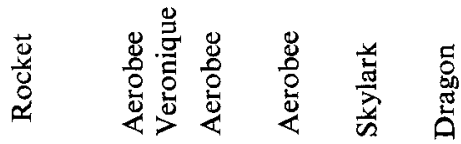

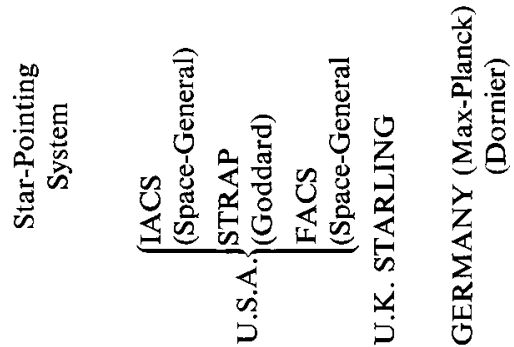


$4000 \AA$ with resolutions of $10 \AA$ and $5 \AA$. Using the same vehicle, Smith (1967b) has flown an objective grating in a Wadsworth mount to obtain a spectrum of Spica to wavelengths below Lyman- $\alpha$. Carruthers (1967) has obtained objective grating spectra with the IACS/Aerobee. An image intensifier of the electronographic type was used to cover the range $950-1400 \AA$ together with two photometers in the respective ranges 1050-1180 $\AA$ and 1230-1350 $\AA$. Courtes and Viton (1967) have used the IACS system in a Veronique rocket to obtain UV sky photographs with an F1 camera in the range $2400-3200 \AA$.

\section{E. POINTING SATELLITES}

Considerable efforts are currently devoted to the development of pointing systems in satellites which will allow the orientation of telescopes to a desired object and subsequent accurate stabilisation for the required period of observation. Such systems are both large and sophisticated, but will present the astronomer with his greatest opportunity for carrying out advanced studies in UV astronomy.

Orbiting Astronomical Observatory $(O A O)$ : This series of satellites is currently under development by NASA and involves a number of UV experiments to be launched sequentially in basically the same spacecraft (Rogerson, 1963). The first of these was launched in April 1966 and although it achieved orbit and stabilisation, a fault in the power sub-system prevented any scientific data being obtained (Space Science Board, 1967). The planned operation is as follows: Firstly, the angular momentum is reduced by using rate gyros and gas jets; then the roll axis is aligned on the Earth-Sun line with the aid of Sun sensors. The observatory is then rotated about the roll axis while the star trackers are set at the correct angles with respect to the programmed reference stars. When these are located the spacecraft control is switched to the star trackers and inertial wheels are used for actuation. The observatory is then slewed to the required object whilst the star trackers continue to track until either occultation occurs or they reach the end of their range. The use of six star trackers ensures the continuous acquisition of sufficient reference stars for the operation of the system.

The OAO experiments are planned in order of increasing spectral resolution. A Wisconsin experiment consists of seven telescopes feeding different forms of photometers, allowing broad-band measurements in the region 1110-3000 $\AA$. A Smithsonian experiment (project Celescope) consists of four telescopes feeding Uvicon image tubes and is designed to map the sky in four wavelength bands centred at $1350 \AA, 1500 \AA$, $2250 \AA$, and $2650 \AA$. Both these experiments require pointing accuracies of the order of 1 arcmin. The Goddard experiment consists of a 36-inch telescope feeding an Eberttype spectrometer used in a scanning mode with six photomultipliers as detectors. The instrument covers the range 1050-4000 $\AA$ with a best spectral resolution of $2 \AA$. The required pointing accuracy of 1 arcsec is to be achieved by the use of an error signal derived from a star sensor within the experimental package. The Princeton experiment covers the range $700-3200 \AA$ and is aimed at a spectral resolution of $0 \cdot 1 \AA$. The 
required pointing accuracy of $0 \cdot 1$ arcsec is also to be achieved with the aid of an experimental error signal. A 32-inch Cassegrain telescope is used over the whole available length in the spacecraft, the spectrometer being spaced between the primary and secondary. The spectrometer is used in a Pachen-Runge mounting and the spectrometer is scanned by two carriages, each containing two exit slits and multipliers. Princeton have also been considering more advanced experiments in the field, and a design study has been carried out by Perkin-Elmer $(1967 a, b)$ to consider the problems involved in orbiting a 40 -inch telescope, operating at its diffraction limit for direct image work and also being used to feed an echelle spectrometer for UV spectral studies with a resolution of $0 \cdot 1 \AA$. One of the many problems associated with this is that of fine stabilisation which is required to 0.01 arcsec and it is proposed to achieve this by magnetically supporting the experimental package within the spacecraft.

European Large Astronomical Satellite ( $L A S$ ): Design studies for a large astronomical satellite to provide a European space observatory have been carried out by ESRO, culminating in a project development plan (ESRO, 1967). This project has not yet been finally approved, but the first experiment has been assigned to spectroscopic studies of objects down to 9th magnitude with variable spectral resolution up to a best value between $0 \cdot 1-0 \cdot 3 \AA$ (Culham Lab., 1966). The LAS concept differs from that of the OAO in that the space reference is derived from gyros. Two star trackers (one for redundancy) are used as a means of up-dating the gyro-reference. An on-board computer will be included to carry out many of the spacecraft functions and it is planned to launch the observatory into an equatorial orbit which gives a minimum of radiation background.

\section{F. MANNED SPACECRAFT}

The first astronomical observations carried out by man in space were performed by U.S. astronauts during the stand-up extra-vehicular activity periods of Gemini flights 10, 11, and 12. The equipment was designed by Henize and Wackerling (1967) and consisted of objective prism and objective grating instruments. Low-dispersion spectra of a number of objects in the range $2300-4000 \AA$ were obtained. An extension of the use of man for astronomical studies in space is currently being considered by NASA. The Apollo Telescope Mount is to be devoted to solar studies and has already been described by Reeves (1968).

\section{G. MOON TELESCOPE}

The possibility of a Moon-based telescope has been considered by Muzjherin et al. (1965) with the aim of establishing a proposal within reach of present-day technology. They suggest a 50-cm meniscus Maksutov telescope to be placed in a vertical position on the lunar surface for UV photometric studies. The rotation of the Moon provides a slow scan of a circle of the sky, a television-type tube being used as detector, and the data being telemetered to Earth. A possible movement of the optical axis from the 
vertical would allow a wider coverage of the sky. It is estimated that such a system could reach magnitudes $8-9$.

\section{Optics}

The optics used in UV astronomy will take several forms but will usually involve a telescope plus ancillary equipment such as spectrometers, photometers, and polarimeters. It is not the intention here to review the whole field of UV optics but to select those developments which are new and linked to a space application either to meet the peculiar environment or the problems associated with operation in the UV.

\section{A. REFLECTING COATINGS}

Great advances have been made during the past decade in the development of highreflecting coatings for use in the UV. A review of these developments and the relevant literature is given by Hunter (1962). Of the several kinds of coatings investigated, five types are particularly valuable for use in $\mathrm{UV}$ astronomy; $\mathrm{Al}+\mathrm{MgF}_{2}, \mathrm{Al}+\mathrm{LiF}, \mathrm{Pt}, \mathrm{Au}$, and $\mathrm{Ge}+\mathrm{ZnS}$. Overcoating of $\mathrm{Al}$ with $\mathrm{MgF}_{2}$ has achieved a reflectivity of about $80 \%$ down to about $1200 \AA$ where it drops steeply. Overcoating of Al with LiF has achieved a reflectivity of about $60-70 \%$ down to about $1000 \AA$. Pt and Au give reflectivities of only $20-30 \%$, but this extends over the full range and they are therefore important for observations down to the Lyman limit. Ge $+\mathrm{ZnS}$ also gives a reflectivity of about $25 \%$ but can give a low reflectivity in the visible for discrimination purposes.

The possibie environmental effects on mirrors coated with $\mathrm{Al}+\mathrm{MgF}_{2}$ have been investigated by Canfield et al. (1966) with encouraging results, reflectivity being maintained after extended exposure to air, UV radiation, and bombardment with $1 \mathrm{MeV}$ electrons and $5 \mathrm{MeV}$ protons. Other relevant information is supplied by Heath and Sacher (1965), who investigated the transmission properties of a number of optical materials after irradiation to 1 and $2 \mathrm{MeV}$ electrons with a dosage of $10^{14}$ electrons $\mathrm{cm}^{-2}$. Whereas a number of materials, including $\mathrm{MgF}_{2}$, were not affected significantly, the transmission of $\mathrm{LiF}$ dropped seriously over a wide range in the $\mathrm{UV}$.

\section{B. OPTICAL MATERIALS}

Operation in a space environment imposes severe requirements on materials used for optical components. This is particularly so for the large primary telescope mirrors being considered for the major observatory satellites, where the optical performance has to be maintained in the face of severe weight restrictions and a peculiar thermal environment. The resulting requirements are:

(1) The material must be good optically, i.e., it must be optically workable to the desired accuracy, must be stable and capable of carrying a high-reflecting coating.

(2) It must have a low weight, i.e., have a high strength/weight ratio, and 
(3) It must be good thermally, i.e., have high conductivity and low expansion.

These requirements are clearly incompatible for existing developed materials. The most essential requirement is (1), since the weight and thermal problems may be solved by other means; e.g., a more powerful rocket booster and a thermostatic thermal design.

For the Princeton OAO experiment, fused silica has been adopted for the telescope mirror. This is a well-tried optical material and the weight problem has been tackled by Corning and Perkin-Elmer by using mirror blanks in an egg crate construction. This gives a mirror whose weight is between $\frac{1}{3}$ and $\frac{1}{2}$ of that of a solid mirror of the same volume. The optical requirement on this particular mirror is for 0.1 arcsec image quality. An easing of the thermal problem may be possible in the future by the use of ultra low-expansion (ULE) silica being developed by Corning (Perkin-Elmer, $1967 a, b$ ), which gives an expansion coefficient of $0.2 \times 10^{-7}{ }^{\circ} \mathrm{C}$ near $20^{\circ} \mathrm{C}$ compared with $4 \times 10^{-7}$ for normal silica. Another low-expansion material, 'Cer-vit', is being developed by Owen (1967) of Illinois and a low-expansion glass, Setal, is being developed in the Soviet Union.

Another approach to this problem is afforded by the use of beryllium. This has a very high strength-to-weight ratio and also has good thermal properties. It presents considerable optical problems, however, some of which have been discussed by Barnes (1966). This material has been adopted for the primary telescope mirror in the Goddard OAO experiment, which has a requirement of 1 arcsec for its image quality. The mirror is coated with Kanigen, an amorphous nickel compound which then carries the high-reflecting coatings.

\section{SPECTROMETERS}

Of the UV payloads discussed above, those which employed dispersing systems have used well-established optical configurations. They are mostly based on concave or plane diffraction gratings and any new major problems posed are mainly of a mechanical or thermal rather than an optical nature. The application of echelle spectrometers will prove valuable in future experiments, since they offer the possibility of high resolution and wide spectral coverage in a compact instrument. Such a system may be used to relieve the pointing accuracy.

Another type of dispersing system which offers advantages in UV astronomy is the Fabry-Pérot etalon which provides high spectral resolution and luminosity, but over a very restricted wavelength range. Since it employs transmission optics its wavelength of operation is restricted by the available optical materials. The shortest wavelength at which such a system has been operated in the laboratory is about $1800 \AA$ where Bradley et al. (1964) used high-quality Spectrosil coated with $\mathrm{Al}+\mathrm{MgF}_{2}$ for the interferometer plates, together with an interference filter consisting of layers of $\mathrm{Al}$ and $\mathrm{MgF}_{2}$ deposited on fused quartz substrates. 


\section{POLARIZERS}

Polarization measurements will become important for certain branches of UV astronomy. The present laboratory position is reasonably good for the range $2000-3000 \AA$ where the use of Calcite and quartz allows polarization measurements to be made with a considerable degree of sophistication. Below $2000 \AA$ polarization devices are in a stage of research and development, but considerable progress has been made in recent years. Burton (1964) constructed a polarization analyzer consisting of a $\mathrm{LiF}$ plate which reflected radiation on the Brewster angle. This simple system is limited in its efficiency and Walker (1964) used a stack of $6 \mathrm{LiF}$ plates giving polarization efficiencies of $82 \%$ at $1200 \AA$ and $65 \%$ at $1600 \AA$ with the respective transmissions of $4.3 \%$ and $21.5 \%$. More sophisticated devices have been developed by Johnson (1964) and Steimetz et al. (1967), who built a Wollaston prism and a double Rochon prism respectively, both in $\mathrm{MgF}_{2}$. The latter device has a transmission greater than $30 \%$ at wavelengths above $1600 \AA$. As a basis for developing both reflecting and transmitting polarizers, Hunter (1964) has studied the optical properties of a number of materials in the UV. The possibility of using thin films has also been considered by Abeles (1964).

\section{Detectors}

\section{A. PHOTOGRAPHIC EMULSION}

The photographic plate is still an important detector in UV astronomy because of its image storage properties and its great simplicity. In addition to its well-known disadvantages as a detector it requires recovery when used in space vehicles. A number of UV emulsions are available of which the fastest are Kodak Pathé SC5 and SC7.

\section{B. PHOTOMULTIPLIER TUBES}

Photomultiplier tubes have now reached an advanced state of development and a wide selection is available commercially which are robust enough for space use and have a wide coverage of spectral response. An extensive literature exists and the reader is referred to the review article by Dunkelman (1962). The types of tube include discrete dynode systems with a variety of photocathodes and windows (Rome, 1964) and magnetic multipliers employing a continuous dynode strip (Heroux and Hinteregger, 1960). The photomultiplier is an excellent detector giving high gain, low noise, and reproducibility. Its only disadvantage for application to UV astronomy lies in its physical size, which limits the number of channels which can be built into any system. 


\section{CHANNEL MULTIPLIERS}

The possibility of very small tubes is offered by the channel multiplier (Goodrich and Wiley, 1961). This consists of a hollow glass cylinder, coated on the inside with a highly resistive material which provides both the continuous dynode and, at the open input end, the photocathode. An electric field applied along the length of the tube causes a cascade of photoelectrons down the tube with subsequent secondary emission. Gains up to $3 \times 10^{8}$ can be achieved (Adams and Manley, 1967) and tube diameters can be as small as fractions of a millimetre. Considerable advances have been made recently by the use of curved tubes which have eliminated the positive ion feed-back produced by electron collisions with residual gas atoms. The open end may be shaped for radiation collection or a slot may be introduced into the side of the tube. The full application of such devices to UV astronomy will require the use of sealed as well as open tubes with a selection of windows and photocathodes. The tubes can also be built in the form of a closely packed channel array giving an imaging system with point by point intensification (Adams and Manley, 1967).

\section{IMAGE STORAGE TUBES}

The image storage tube combines the major advantages of the photographic plate and photomultiplier tube. Its imaging and storage properties lead to a large information capacity where the electrical characteristic of the signals allows the telemetry of the information and hence its use in satellites. Considerable effort is currently under way for the development of such devices for application over a very wide range of fields and the requirements for their application to UV astronomy are particularly demanding. These requirements can be listed as follows:

(1) A spectral response covering most and preferably all of the wavelength range $900-3000 \AA$.

(2) A long storage time - from minutes up to several hours depending on the particular use.

(3) A low background to permit long integration times.

(4) A high spatial resolution.

(5) Good reproducibility.

(6) Compatability with spacecraft.

(7) Low weight.

(8) Low power consumption.

(9) Rugged design.

A review of the possible application of image storage tubes to UV astronomy has been made by Boksenberg (1965) and the discussion here will be limited to two devices which have either been flown or specifically assigned to a space experiment. The first is not an electric image tube in the full sense, but is an image intensifier of the elec- 
tronographic (Lallemand) type flown by Carruthers in a pointing Aerobee to obtain objective grating stellar spectra (Carruthers, 1967). The tube (Carruthers, 1965) uses a $\mathrm{KBr}$ photocathode with magnetic focussing and an accelerating potential of $20 \mathrm{kV}$, the electron image being focussed to a nuclear emulsion. The second device, known as the Uvicon (Skorinko et al., 1961) is being developed by Westinghouse Research Laboratories for the Smithsonian OAO experiment (Project Celescope) (Davis and Rustgi, 1962). The tube is sealed and the imaging and read-out sections are entirely electrostatic. The original tube has been modified to incorporate an SEC (secondary electron conduction) target (Boerio et al., 1966) which is capable of storage times of $\sim 24$ hrs. (Doughty, 1966). Tubes with various spectral responses through the ultraviolet are obtained by suitable selection of window and photocathode.

\section{References}

Abeles, F. (1964) Proc. Conf. Photo. Spec. Optics (Tokyo and Kyoto), p. 517.

Adams, J., Manley, B.W. (1967) Philips Tech. Rev., 28, 156.

Alexander, J.D. H., Bowen, P.J., Heddle, D.W.O. (1964) Proc. Roy. Soc., A279, 510.

Barnes, W.P. (1966) Appl. Opt., 5, 1883.

Boerio, A.H., Beyer, R.R., Goetze, G.W. (1966) Adv. Electronics Electron. Phys., 22A, 229.

Boggess, A. (1961) Mem. Soc. Roy. Sci. Liege, 4, 459.

Boggess, A. (1964) IAU Symposium No. 23, Liège, p. 173.

Boggess, A. (1967) IAU Symposium on Space Spectroscopy, Dearborn.

Boksenberg, A. (1965) ESRO Memorandum SM-5.

Bradley, D.J., Bates, B., Juulman, C.O.L., Majamdar, S. (1964) Nature, 202, 579.

Burton, W. M. (1964) Internal Culham Memorandum.

Byram, E.T. et al. (1957) Astron. J., 62, 9.

Byram, E.T., Chubb, T.A., Werner, W.M. (1964) IAU Symposium No. 23, Liège, p. 177.

Canfield, L.R., Hass, G., Waylonis, J.E. (1966) Appl. Opt., 5, 45.

Carruthers, G.R. (1965) Report to NRL Progress, July.

Carruthers, G.R. (1967) IAU Symposium on Space Spectroscopy, Dearborn.

Chubb, T. A., Byram, E. T. (1963) Astrophys. J., 138, 617.

Courtes, G., Viton, M. (1967) Paper 96, CoSPAR Meeting, London.

Culham Laboratory (1966) Design Study for Scientific Package in European Large Astronomical Satellite.

Davis, R.J., Rustgi, O.P. (1962) Appl. Opt., 1, 131.

De Jager, C., Underhill, A. B., Emming, J. G., Hammerschag, A.(1967) Tech.Phys. Dienst. Report No. 609, 502.

Dimov, N.A., Severny, A.B. (1965) Proc. Int. Astronautical Congress (Athens).

Doughty, D.D. (1966) Adv. Electronics Electron. Phys., 22A, 261.

Dunkelman, L. (1962) J. quantit. Spectrosc. Radiat. Transfer, 2, 533.

Elliott Bros. (1967) Report $T(F) 68$.

ESRO (1967) Project Development Plan for the Large Astronomical Satellite - A European Space Observatory.

Friedman, H. (1962) J. quantit. Spectrosc. Radiat. Transfer, $2,547$.

Golay, M., Gaide, A., Huguenin, D. (1967) IAU Symposium on Space Spectroscopy, Dearborn. Goodrich, G.W., Wiley, W.C. (1961) Proc. Image Intensifier Symp. Fort Belvoir, Va., U.S.A.

Gulledge, I.S., Packer, D. M. (1963) Astron. J., 68, 537.

Heath, D.F., Sacher, P. A. (1965) Goddard Report X-622-65-382.

Henize, K., Wackerling, L. (1967) IAU Symposium on Space Spectroscopy, Dearborn.

Heroux, L., Hinteregger, H.E. (1960) Rev. Sci. Instrum., 31, 280. 
Housiaux, L. (1965) Second Report Edinburgh/Liège Scanning Experiment, Inst. d'Astrophys., Liège.

Hunter, W.R. (1962) Optica Acta, 9, 255.

Hunter, W.R. (1964) Proc. Conf. Photo. Spec. Optics (Tokyo and Kyoto), p. 520.

Johnson, W.C. (1964) Rev. Sci. Instrum., 35, 1375.

Kurt, V.G., Syunyaev, R.A. (1967) JETP Letters, 5, 246.

Morton, D.C. (1967) Astrophys. J., 147, 1017.

Morton, D.C., Spitzer, L. (1966) Astrophys. J., 144, 1.

Muzjherin, V.M., Nikonov, V.B., Prokofiev, V.K., Chernykh, N.S. (1965) Proc. Int. Astronautical Congress (Athens).

NASA (1967) Document NHB8030.1A, Opportunities for Participation in Space Flight Investigations.

Owen, G. (1967) Sky and Telescope, 34, 293.

Perkin-Elmer (1967a) Report No, 8346(I), Princeton Advanced Satellite Study.

Perkin-Elmer (1967b) Report No. 8688, Princeton Advanced Satellite Study.

Reeves, E.M. (1968) in the present volume, p. 75.

Rogerson, J. B. (1963) Space Sci. Rev., 2, 621.

Rome, M. (1964) IEEE Trans. Nucl. Sc. NS-11, 93.

Skorinko, G., Doughty, D.D., Feibelman, W.A. (1961) Westinghouse Res. Lab. Sci. Paper 912-J902-01.

Smith, A.M. (1967a) Astrophys. J., 147, 158.

Smith, A.M. (1967b) Report on Commission 44, IAU Meeting, Prague.

Space Science Board, Nat. Res. Council (1967) U.S. Space Science Programme, Report to COSPAR, London.

Stecher, T.P. (1967) IAU Symposium on Space Spectroscopy, Dearborn.

Stecher, T.P., Milligan, J.E. (1962) Astrophys. J., 136, 1.

Steimetz, D.L., Phillips, W.G., Winick, M., Forbes, F.F. (1967) Appl. Opt., 6, 1001.

Walker, W.C. (1964) Appl. Opt., 3, 1457.

\section{DISCUSSION}

D.C. Morton: In discussing the possibility of putting optical instruments on the Moon, one fact that we should always take into consideration is the great disadvantage of the Moon's gravitational field with all the old problems of flexure.

$R$. Wilson: What you say is true, of course. It is also true that if you put a man into a large orbiting laboratory you introduce a certain increased cost, and also a certain increase in the complexity of operation. I'm not sure whether the kind of impartial analysis which would be required has really been made in the full sense of evaluating all these different techniques involving man, the Moon, sounding rockets, and unmanned satellites. It is a very difficult assessment to make.

L. Gratton: Could you mention the possibility of an automatic telescope visited infrequently by astronauts?

R. Wilson: Yes. There is the combination of approaches, using the astronaut as the repair agent for an unmanned experiment.

G.J. Odgers: What would be required to do diffraction limited stellar spectroscopy from above the Earth's atmosphere with a line stabilization of the order of hundredths of a second of arc? What are the engineering possibilities, and how does the stabilization compare with the best so far achieved?

$R$. Wilson: This particular proposal is an advanced one which is not entirely accepted by NASA. A study was carried out by the Perkin-Elmer Corporation for Princeton University in which they reached the conclusion that such stabilization was feasible. They proposed to have magnetic stabilization in which the instrument package is mechanically separated from the spacecraft. There are very attractive advantages to this system, because one of the great problems of an astronomical satellite is the experiment-spacecraft interface. If you can have this separation, then the thermal interface problems improve tremendously.

R.E. Danielson: I would like to add something on the achievement of one arc second pointing. 
The biggest problem there is to sense the error since when you have a diffraction-limited telescope you have images which are a tenth of an arc second in diameter. The difficult problem is the thermal problem that you mentioned.

G. A. Newkirk, Jr.: I would like to comment on the problem of maintaining a fairly large aperture optical system in diffraction limit. There is a program now in progress at the Perkin-Elmer Corporation on 'active optics'. The mirror is either composed of several pieces, or one has a large mirror which is rather flexible. One senses the figure of this mirror by an auxiliary system which encircles the mirror and adjustments are made to maintain the appropriate figure. They have just begun and now have a working model for a telescope about 12 inches in diameter. 